\title{
Hydrogen abundance estimation model and application to (162173) Ryugu
}

\author{
A. Praet ${ }^{1}$, M. A. Barucci ${ }^{1}$, P. H. Hasselmann ${ }^{1}$, K. Kitazato ${ }^{2}$, T. Iwata ${ }^{3}$, M. Matsuoka ${ }^{3}$, \\ D. Domingue ${ }^{4}$, and B. E. Clark \\ ${ }^{1}$ LESIA, Observatoire de Paris, PSL Research University, CNRS, Univ. Paris Diderot, Sorbonne Paris Cité, UPMC Univ. Paris 06, \\ Sorbonne Universités, 5 place Jules Janssen, 92195 Meudon, France \\ e-mail: alice.praet@obspm.fr \\ 2 The University of Aizu, Aizu-Wakamatsu 965-8580, Japan \\ 3 ISAS, JAXA, Sagamihara 252-5210, Japan \\ 4 Planetary Science Institute, Tucson, AZ 85710, USA \\ 5 Department of Physics, Ithaca College, Ithaca, NY 14850, USA
}

Received 26 March 2021 / Accepted 16 April 2021

\section{ABSTRACT}

\begin{abstract}
Context. The JAXA asteroid sample return mission Hayabusa2 acquired a huge quantity of data from the asteroid (162173) Ryugu during its 1.5 years in asteroid proximity orbit. On December 5, 2020 (Japan time), Hayabusa brought back to Earth a 5.4 g sample from Ryugu's surface.

Aims. We analyzed the near-infrared spectra of Ryugu, in particular the band at $2.72 \mu \mathrm{m}$, with the aim to investigate the hydrogen content of the $\mathrm{H}_{2} \mathrm{O}$ and $\mathrm{OH}^{-}$groups in hydrated phyllosilicates on Ryugu's surface.

Aims. We applied two different methods, normalized optical path length (NOPL) and effective single-particle absorption thickness (ESPAT), to the $3 \mu \mathrm{m}$ region absorption band, and we compared the obtained spectral parameters with those obtained from carbonaceous chondrite meteorites whose $\mathrm{H}$ content was determined in the laboratory.

Methods. We derived an exponential correlation between the selected meteorite $\mathrm{H}$ content and its respective ESPAT and NOPL parameters. The average value of the $\mathrm{H}$ content obtained on Ryugu's surface with its relative variations, combining the results obtained with the two methods, is $0.52_{-0.21}^{+0.16}$ wt.\%. These methods can be applied to other asteroids that exhibit a $3 \mu \mathrm{m}$ region absorption band to estimate the mean average of $\mathrm{H}$ content.

Results. The results of the ESPAT and NOPL methods used on the Ryugu spectral data present small variations across Ryugu's surface and do not show any evident relation with the surface geomorphological structures. Our estimation of the global average $\mathrm{H}$ content of Ryugu is in agreement with those of several aqueously altered carbonaceous chondrites measured in the laboratory and is most similar to the $\mathrm{H}$ content of heated CM. The study of phyllosilicate $\mathrm{H}_{2} \mathrm{O}$ and $\mathrm{OH}^{-}$group hydrogen content on Ryugu and the derived method may be applied to other observed primitive asteroids. The obtained results will allow Solar System evolution models to be constrained and will allow the formation and evolution of the Solar System to be better understood.
\end{abstract}

Key words. minor planets, asteroids: individual: (162173) Ryugu - methods: statistical - methods: data analysis techniques: spectroscopic - meteorites, meteors, meteoroids

\section{Introduction}

In late June 2018, Hayabusa2, the Japanese Aerospace Exploration Agency (JAXA) spacecraft for the asteroid sample return mission, arrived at home position (HP), located at an altitude of about $20 \mathrm{~km}$ from Ryugu's surface, in a hovering position orbit. It acquired a large quantity of data of the near-Earth asteroid (162173) Ryugu using several instruments: the Optical Navigation Camera Telescope (ONC-T), a thermal infrared imager (TIR), a near-infrared spectrometer (NIRS3), and a laser light detection and ranging (LIDAR) system (Watanabe et al. 2019).

The onboard instruments revealed a very dark surface object with a geometric albedo of $0.045 \pm 0.02$ (Sugita et al. 2019), a mean equatorial diameter of $1.004 \pm 0.004 \mathrm{~km}$, and a rotational period of $7.63262 \pm 0.00002 \mathrm{~h}$ (Watanabe et al. 2019). Ryugu showed a top-shape form with a polar-to-equatorial-axis ratio of $0.872 \pm 0.007$ and a very low density, which could imply a rubble-pile nature (Watanabe et al. 2019). The camera images revealed many geomorphological features, such as craters, ridges, depressions, and numerous boulders of different sizes, the largest of which (Otohime Saxum) was about $160 \mathrm{~m}$ in diameter (Sugita et al. 2019).

The NIRS3 instrument, operating in scanner mode, acquired reflectance spectra from 1.8 to $3.2 \mu \mathrm{m}$ (Iwata et al. 2017). The NIRS3 is a point spectrometer with a $0.1^{\circ}$ field-of-view, which obtained continuous point-target spectra over Ryugu's surface. The obtained spectra show a weak narrow absorption band at $2.72 \mu \mathrm{m}$, which was detected across all of the observed surface. The absorption band indicates the presence of $\mathrm{OH}$-bearing minerals, associated with $\mathrm{Mg}$-rich phyllosilicates (Kitazato et al. 2019) that are ubiquitously present on the surface of Ryugu. No meteoritic samples with spectra available at NIRS3 wavelengths seem to fully match those of Ryugu, but there are similarities 


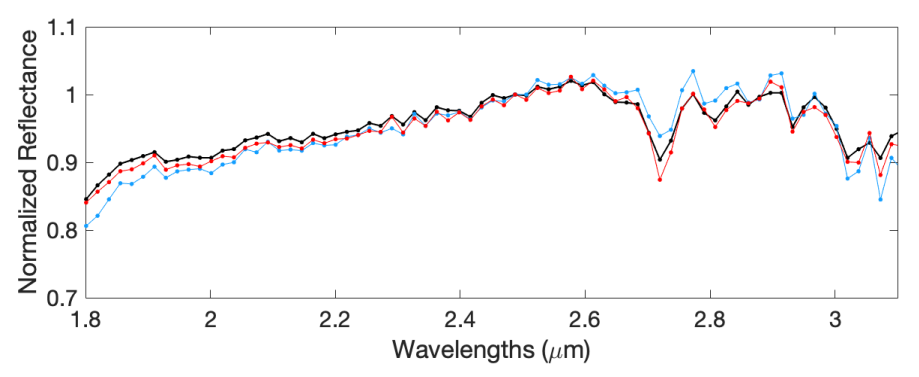

Fig. 1. Average normalized (at $2.5 \mu \mathrm{m}$ ) Ryugu spectrum from July 19, 2018 (in black), and the spectra showing the deepest and shallowest $2.72 \mu \mathrm{m}$ band depth (in red and blue, respectively), using a linear continuum from 2.60 to $2.90 \mu \mathrm{m}$.

with the spectra of thermally metamorphosed CI and shocked CM chondrites (Kitazato et al. 2019).

The spectral data of Ryugu show an almost homogeneous surface with only small variations (Kitazato et al. 2019; Sugita et al. 2019). Barucci et al. (2019) analyzed the spectrophotometric and spectral data via multivariate statistical analysis and detected a small spectral slope variation, which they interpreted as a consequence of space weathering, and the presence of more or less fresh material on the surface of Ryugu.

In the present work, we apply the effective single-particle absorption thickness (ESPAT; Milliken \& Mustard 2007a,b) and normalized optical path length (NOPL; Milliken \& Mustard 2007a,b; Garenne et al. 2016) methods to the $2.72 \mu \mathrm{m}$ absorption band of the Ryugu spectral data obtained by the Hayabusa2 mission. These methods were already applied to spectral data analyses of Mars (Mustard et al. 2008) and the Moon (Li \& Milliken 2017) and were used to analyze the surface of Bennu (Praet et al. 2021). We aim to compare these two spectral parameters, which were computed on carbonaceous chondrite spectra data, with the meteorite $\mathrm{H}$ content; we also aim to derive Ryugu's surface mean $\mathrm{H}$ content from the two meteorite correlations and from Ryugu's mean spectral parameter values.

\section{Data analysis}

\subsection{Ryugu data}

For the NIRS3 data, we used the spectra collected on July 19, 2018, which allowed us to obtain near-global coverage from $-70^{\circ}$ to $+70^{\circ}$ latitudes. These observations were carried out at phase angles of $17.5-17.8^{\circ}$ at a spatial resolution of $20 \mathrm{~m} / \mathrm{px}$. The NIRS3 data were calibrated and photometrically corrected using the Hapke model (Kitazato et al. 2019) to the standard viewing geometry of $\left(30^{\circ}, 0^{\circ}, 30^{\circ}\right)$ for the incidence, emergence, and phase angles, respectively. The average spectrum of our data set is shown in Fig. 1, as are spectra characterized by the shallowest and deepest $2.72 \mu \mathrm{m}$ absorption band depth. To avoid data that are not well corrected near the poles, we limited our analysis to $-60^{\circ}$ to $+60^{\circ}$ latitudes, as reported in the maps in Fig. 2. The variations in the spectra are very small and essentially concern the slope between $2.00 \mu \mathrm{m}$ and $2.60 \mu \mathrm{m}$ (and to a lesser extent the band depth at $2.72 \mu \mathrm{m}$ ), with no variation in the position of the band minimum.

\subsection{Meteorites}

We gathered laboratory-measured carbonaceous chondrite reflectance data from Potin et al. (2019), Takir et al. (2013, 2019), and Garenne et al. (2016). We selected 43 high quality reflectance spectra from a total of 30 carbonaceous chondrites measured under asteroid-like conditions: fifteen CMs, eight heated CMs, two CIs, two CVs, two CRs, and Tagish Lake. They each exhibit an absorption band in the $3 \mu \mathrm{m}$ region diagnosis of $\mathrm{H}_{2} \mathrm{O}$ - and/or OH-bearing hydrated phyllosilicates (Appendix A), and their bulk compositions have been independently measured in the laboratory by Alexander et al. $(2012,2013)$. The hydrogen content of the hydrated phyllosilicate $\mathrm{H}_{2} \mathrm{O}$ and $\mathrm{HO}^{-}$groups (i.e., $\mathrm{H}$ content) of each meteorite was then derived from its bulk composition (bulk carbon and bulk hydrogen contents) following the method described by Alexander et al. (2012). Assuming that all carbon is contained in the meteorite organic matter, we estimated the organic hydrogen content using the bulk carbon content multiplied by the hydrogen-to-carbon ratio $(\mathrm{H} / \mathrm{C})$ of typical $\mathrm{CM}$ insoluble organic matter (IOM) for CM meteorites and of CR IOM for the remaining carbonaceous chondrites. Subtracting the organic hydrogen content from the bulk hydrogen content, the hydrogen content of the hydrated phyllosilicates (contained in their water and hydroxyl groups) can be estimated. The $\mathrm{H}$ content of Tagish Lake lithology 4 and WIS91600 are from Gilmour et al. (2019) and Garenne et al. (2016), respectively (Appendix A). In cases where several $\mathrm{H}$ content values are available in the literature due to the multiple lithologies of a meteorite, we chose to take all $\mathrm{H}$ content values into account.

\subsection{Method}

We applied two different methods to estimate Ryugu's surface H content. Two distinct spectral parameters, ESPAT and NOPL, were computed on Ryugu spectra as well as on laboratorymeasured meteorite reflectance spectra. Both sets of meteorite spectral parameter results were then compared to the spectra's composition data, that is, the hydrogen content of the $\mathrm{H}_{2} \mathrm{O}$ and $\mathrm{HO}^{-}$groups in hydrated phyllosilicates (Alexander et al. 2012, 2013). Finally, we fit a regression model that enabled us to derive the average hydrogen content of the $\mathrm{H}_{2} \mathrm{O}$ and $\mathrm{HO}^{-}$groups in the hydrated phyllosilicates of Ryugu's surface using the previously calculated spectral parameters of Ryugu's spectra.

Normalized Optical Path Length (NOPL). The NOPL $(\lambda)$ parameter was calculated as described in Garenne et al. (2016), Milliken \& Mustard (2005), and Milliken et al. (2007) for each meteorite spectrum and for each individual absolute reflectance $(R)$ spectrum of Ryugu. A linear continuum $\left(R_{c}\right)$ was fitted from 2.6 to $2.9 \mu \mathrm{m}$. The NOPL parameter was calculated at the wavelength center $(\lambda)$ of $2.72 \mu \mathrm{m}$, which is the mean band minimum position for the data set (Kitazato et al. 2019). Three wavelength channels were averaged for both continuum anchor points; however, a single wavelength channel was used for the center point in order to avoid an underestimation of the calculated parameter since the band minimum position is constant throughout the studied spectral data set. The NOPL parameter equation is:

$\operatorname{NOPL}(\lambda)=\frac{-\ln \left(R(\lambda)+1-\ln \left(R_{c}(\lambda)\right)\right)}{-\ln \left(1-\ln \left(R_{c}(\lambda)\right)\right)}$

Effective Single-Particle Absorption Thickness (ESPAT). The $\operatorname{ESPAT}(\lambda)$ parameter was calculated following the method in Garenne et al. (2016; see also Milliken \& Mustard 2005; Milliken et al. 2007). The absolute reflectance spectra of meteorites and Ryugu's I/F spectra surface were first converted into single-scattering albedo $(w(\lambda)$, or $S S A(\lambda))$ spectra (Garenne et al. 2016; Milliken \& Mustard 2005; Milliken et al. 2007) using the Ryugu surface Hapke global parameter from Domingue et al. (2021). A linear continuum $\left(w_{\max }(\lambda)\right)$ was then 

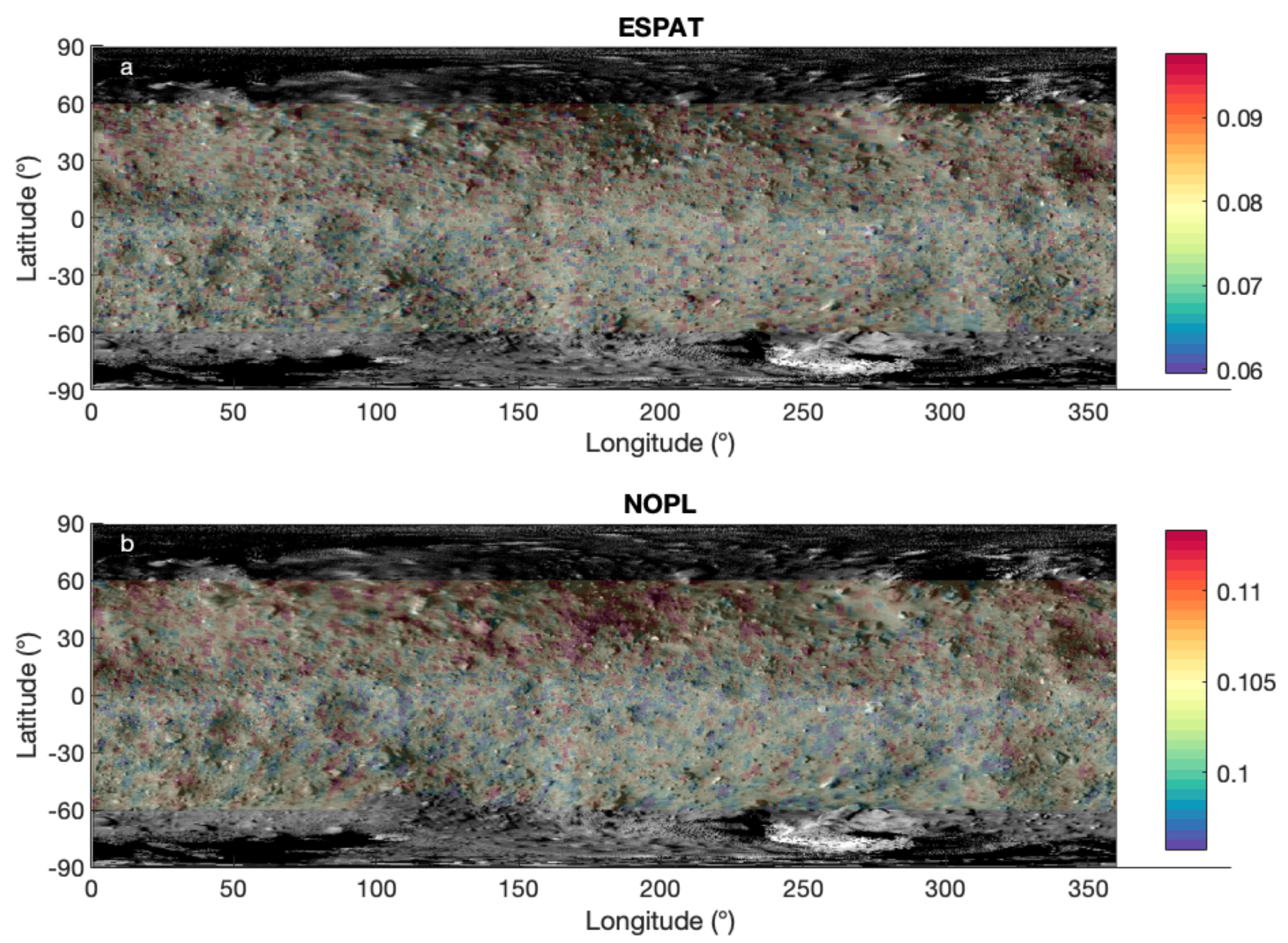

Fig. 2. ESPAT (panel $a$ ) and NOPL (panel $b$ ) parameters (limited to $-60^{\circ}$ to $60^{\circ}$ latitudes) calculated on Ryugu spectra collected on July 19 , 2018, mapped on the Ryugu global base map. They are calculated at the center wavelength of $2.72 \mu \mathrm{m}$ and using a linear continuum from 2.60 to $2.90 \mu \mathrm{m}$.

fit from 2.6 to $2.9 \mu \mathrm{m}$, and the ESPAT parameter was calculated at $2.72 \mu \mathrm{m}(\lambda)$ as well. The numbers of wavelength channels considered for the continuum anchor point and center point are the same as for NOPL. The ESPAT parameter was calculated using the volume-averaged single-scattering albedo $(w(\lambda))$ as follows:

$$
\operatorname{ESPAT}(\lambda)=\frac{1-\frac{w(\lambda)}{w_{\max }(\lambda)}}{\frac{w(\lambda)}{w_{\max }(\lambda)}} .
$$

In the case of the meteorites, both spectral parameter linear continuum anchor point wavelengths were adapted for each meteorite; they are reported in Appendix A.

These two methods were used by Milliken \& Mustard (2005, $2007 \mathrm{a}, \mathrm{b})$ to determine the water content of minerals using laboratory-measured reflectance spectra. Pommerol \& Schmitt (2008a,b) studied the effects of reflectance spectrum, viewing geometry as well as meteorite particle size on the $3 \mu \mathrm{m}$ region $\mathrm{H}_{2} \mathrm{O}$ absorption band (not taking the hydroxyl absorption near $2.7 \mu \mathrm{m}$ into account) for two hydrated minerals. The spectral parameters computed were, among others, NOPL and ESPAT. They found large variations in the band depth to be present in the case of large phase angles.

Mapping. For the NOPL parameter, the photometrically corrected reflectance spectra (collected on July 19, 2018) on which the calculations were performed were averaged using, as the weight, the footprint coverage of the shape model facet over the 49152 facets of Ryugu's shape model version from August 10, 2018 (S HAPE_S PC_49K_V20180810). The ESPAT parameter was calculated on single-scattering albedo spectra that were derived from I/F spectra, which included 21607 spectra in total. The ESPAT symbol size used for the map (Fig. 2a) was adapted to fit the spatial resolution of $20 \mathrm{~m} / \mathrm{px}$ of the studied spectral set. Both Ryugu spectral parameter maps were finally overlaid on the asteroid's surface global base map to look for any correlation between the calculated spectral parameters and the asteroid's surface geomorphology.

\section{Results}

\subsection{Ryugu}

The mapped parameters ESPAT and NOPL computed on Ryugu's spectra are reported in Figs. $2 \mathrm{a}$ and b, respectively. The ESPAT and NOPL parameter variations are very small and do not show any correlation with the surface morphology. The analysis of our data does not show any dichotomy for the ESPAT and NOPL parameters between the eastern and western hemispheres, unlike what was detected by Sugita et al. (2019) on the basis of geomorphological features and spectral colors.

The distributions of the computed ESPAT and NOPL meteorite values versus their $\mathrm{H}$ content are presented in Figs. 3a 

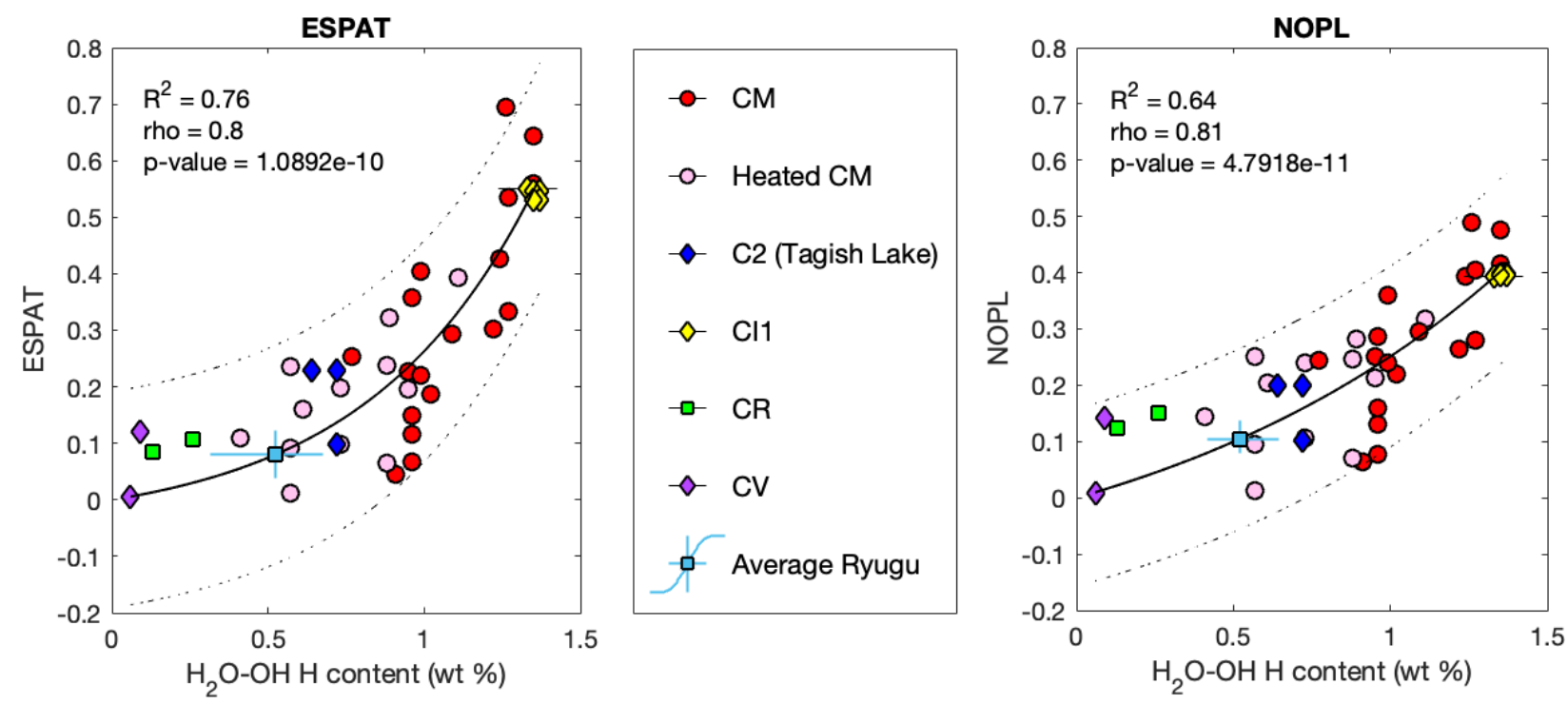

Fig. 3. Exponential correlation between the meteorite $\mathrm{H}$ content (Appendix A) and its ESPAT parameter (left) and NOPL parameter (right), including different types of hydrated CC meteorites as well as Ryugu. Parameter errors for the meteorites are smaller than the symbols. The thick black line is the exponential regression of the meteorite data; the two dashed black lines define the prediction bounds, which indicate the area in which a new data point would fall with a 95\% probability. The mean $\mathrm{H}$ content values for Ryugu, determined using the meteorite exponential correlations, are $0.52 \mathrm{wt} . \%$ in both cases. Ryugu H content relative variation (horizontal bars; $0.313 \mathrm{wt} . \%-0.675 \mathrm{wt}$. $\%$ using ESPAT and $0.415 \mathrm{wt} . \%-0.643 \mathrm{wt} . \%$ using NOPL) is computed using the minimum and maximum values of the ESPAT and NOPL spectral parameters (vertical bars), respectively.

and $b$, respectively. Lower $\mathrm{H}$ content meteorites are characterized by low NOPL as well as low ESPAT, and vice versa. We find that an exponential-type regression curve best fits the data obtained with the two methods. The exponential equation is:

$y=a \cdot\left(e^{b \cdot x}-1\right)$,

where $a$ and $b$ are coefficients determined using the least-squares method, which minimizes the summed squares of the residuals. The regression is also forced to pass through point $(0,0)$, as in the case of an ESPAT or NOPL null (i.e., no $3 \mu \mathrm{m}$ region absorption band and therefore no hydrated phyllosilicates), and as such the $\mathrm{H}$ content in the hydrated phyllosilicates is also null. To assess the quality of the regression fit on each of the correlations, we computed the Spearman correlation, which consists of a pair of coefficients: rho and p-value, reported in Figs. 3a and b. Both rho values are higher than 0.5 , and their associated $p$-value are less than 0.05 , which indicates a strong correlation in both cases. The $R^{2}$ coefficient was also computed for both fit exponential regressions and is higher than 0.5 in both cases $(0.76$ and 0.64 for the ESPAT and NOPL regression fits, respectively), which proves that they are both good quality regressions and that the ESPAT regression is of slightly higher quality.

The mean values of Ryugu's $\mathrm{H}$ content were determined by using the meteorite exponential correlations (Figs. 3a and b). The two dashed black lines visible in Figs. $3 a$ and $b$ define the prediction bounds, which indicate the area in which a new data point would fall with a $95 \%$ probability. The vertical bar on Ryugu's mean value is the minimum and maximum values of the ESPAT and NOPL spectral parameters. Ryugu mean $\mathrm{H}$ content variation (horizontal bars in Figs. 3a and b) were computed for each method using Ryugu minimum and maximum ESPAT and NOPL parameter values.

The mean $\mathrm{H}$ content and its variation, computed for Ryugu with the two methods, are reported in Table 1. The two different methods give similar results, confirming their quality, and allow for a global mean $\mathrm{H}$ content of Ryugu's surface and its average relative variations of $0.52_{-0.21}^{+0.16} \mathrm{wt}$. $\%$. The surface $\mathrm{H}$ content variation was computed using the minimum and maximum values of the ESPAT and NOPL spectral parameters and averaging their results.

\subsection{Model}

On the basis of our analysis, we propose two simple models to be used for an approximation of the asteroid $\mathrm{H}$ content estimation ( $H$ in Eqs. (4) and (5)). Two equations were derived using the ESPAT mean parameter $(E p)$ and the NOPL mean parameter $(N p)$, reported in Eqs. (4) and (5), respectively, with the coefficients $a$ and $b$ derived from their respective best exponential regression fit:

$H=0.540 \cdot\left(\ln \left(\frac{E p}{0.050}\right)+1\right)$,
$H=1.196 \cdot\left(\ln \left(\frac{N p}{0.192}\right)+1\right)$.

These models can be applied to other primitive hydrated phyllosilicate-bearing asteroids with a fully observed $3 \mu \mathrm{m}$ region absorption band to obtain a mean estimation of the $\mathrm{H}$ content of phyllosilicate $\mathrm{H}_{2} \mathrm{O}$ and $\mathrm{OH}^{-}$groups.

The obtained models obviously only represent approximations as it is well known that the $3 \mu \mathrm{m}$ region absorption band strength varies depending on a number of parameters, such as morphological properties (grain size and porosity), surface temperature, and geometric illumination (Potin et al. 2019; Pommerol \& Schmitt 2008a,b). In our case, we obtained similar results from both methods. We suggest that it is generally preferable to use the NOPL method (derived directly from reflectance spectra), particularly for telescopic ground observations, as it has the advantage of not requiring conversion to single-scattering 
Table 1. Summary of ESPAT and NOPL spectral parameter values and the derived $\mathrm{H}$ content.

\begin{tabular}{lcccc}
\hline \hline Spectral parameter & $\begin{array}{c}\text { Spectral parameter } \\
\text { mean value }\end{array}$ & $\begin{array}{c}\text { Spectral parameter } \\
\text { min-max range }\end{array}$ & $\begin{array}{c}\text { H content } \\
\text { mean (wt. \%) }\end{array}$ & $\begin{array}{c}\text { H content } \\
\text { min-max range (wt.\%) }\end{array}$ \\
\hline ESPAT & 0.078 & $0.039-0.122$ & $\mathbf{0 . 5 2 3}$ & $\mathbf{0 . 3 1 3 - 0 . 6 7 5}$ \\
NOPL & 0.105 & $0.080-0.137$ & $\mathbf{0 . 5 2 1}$ & $\mathbf{0 . 4 1 5 - 0 . 6 4 3}$ \\
\hline
\end{tabular}

albedos, which may add uncertainties. But if the user is able to accurately convert spectra to single-scattering albedos, the ESPAT becomes more interesting as it is suppressed from surface photometric effects.

These estimations of the $\mathrm{H}$ content on spectrally observed asteroids may be underestimated regardless of the method used. In fact, Beck et al. (2021), comparing the spectral parameters (sum of band depths at 2.75 and $2.8 \mu \mathrm{m}$ ) calculated on Main Belt asteroids observed by AKARI with those of carbonaceous chondrites, found that the water content estimated for the hydrated asteroids is lower than that measured for carbonaceous chondrites.

\section{Conclusions}

The understanding of the origin of water on Earth is one of the most important topics still debated (Alexander et al. 2012; Altwegg et al. 2015; Piani et al. 2020). Many primitive asteroids once contained abundant water, which is now stored as $\mathrm{OH}$ in hydrated minerals (Alexander et al. 2012). For this reason, we investigated the band at $2.72 \mu \mathrm{m}$ in depth with the aim to estimate the hydrogen content of the $\mathrm{H}_{2} \mathrm{O}$ and $\mathrm{HO}^{-}$groups in the hydrated phyllosilicates on the surface of Ryugu.

Our prime result gives the estimation of the global mean $\mathrm{H}$ content value on Ryugu' surface of $0.52_{-0.21}^{+0.16} \mathrm{wt} \%$. The estimated $\mathrm{H}$ content variation is not connected with Ryugu's surface morphology, nor are the Ryugu ESPAT and NOPL parameters. The derived $\mathrm{H}$ content is in agreement with those of several aqueously altered carbonaceous chondrites measured in the laboratory and is most similar to the $\mathrm{H}$ content of heated CM. We expect to confirm these results with the analysis of return regolith samples from Ryugu. On December 5, 2020 (Japan time), Hayabusa2 successfully brought to Earth a sample from the surface of Ryugu. The detailed analysis of this sample will shed light on water and organic contents, which are important elements for our understanding of the origin of the Solar System.

The described NOPL and ESPAT methods were applied to asteroids for the first time using the spectral data of the primitive near-Earth asteroid Bennu, which was visited by NASA's OSIRIS-REx space mission (Praet et al. 2021). In the present work, we derived an exponential correlation of the $\mathrm{H}$ content measured on meteorites that can be used as a method to derive $\mathrm{H}$ content estimations for other primitive objects. The user may choose one of the two functions depending on the method most suitable for the available data set. In our case, both methods give very similar results, with a slightly better approximation with the ESPAT correlation, in agreement with Milliken \& Mustard (2007b) and Pommerol \& Schmitt (2008a).
The estimation of the phyllosilicate $\mathrm{H}_{2} \mathrm{O}$ and $\mathrm{HO}^{-}$hydrogen content on Ryugu, if confirmed via laboratory analysis on the returned sample, will allow the application of the derived models to other asteroids that exhibit a $3 \mu \mathrm{m}$ absorption band to estimate their mean $\mathrm{H}$ content. The study of $\mathrm{H}_{2} \mathrm{O}$ and $\mathrm{HO}^{-}$ abundances on primitive asteroids is important for understanding the origin of water and can allow us to constrain models and dynamical processes to better understand the origin and evolution of our Solar System. It will help toward settling the controversy that surrounds the origin, abundance, and history of water on Earth as well as better understanding the key processes in the development of life on Earth (Brack 1993).

Acknowledgements. We thank the Hayabusa2 JAXA teams for their efforts in making the mission successful. Hayabusa2 was developed and built under the leadership of JAXA, with contributions from the DLR and the CNES, and in collaboration with NASA, Nagoya University, University of Tokyo, National Astronomical Observatory of Japan, Aizu University, Kobe University, and other universities, institutes, and companies in Japan. We would also wish to thank all the engineers who contributed to the success of Hayabusa 2 mission. A. P., M. A. B., and P. H. H. acknowledge funding support from CNES.

\section{References}

Alexander, C. M. O.’D., Bowden, R., Fogel, M. L., et al. 2012, Science, 337, 721

Alexander, C. M. O.'D., Howard, K. T., Bowden, R., \& Fogel, M. L. 2013, Geochim. Cosmochim. Acta, 123, 244

Altwegg, K., Balsiger, H., Bar-Nun, A., et al. 2015, Science, 347, 387

Barucci, M. A., Hasselmann, P. H., Fulchignoni, M., et al. 2019, A\&A, 629, A13

Beck, P., Eschrig, J., Potin, S., et al. 2021, Icarus, 357, 14125

Brack, A. 1993, Origins Life Evol. Biosphere, 23, 3

Domingue, D., Kitazato, K., Matsuoka, M., et al. 2021, Plan. Sci. J., submitted

Garenne, A., Beck, P., Montes-Hernandez, G., et al. 2016, Icarus, 264, 172

Gilmour, C. M., Herd, C. D. K., \& Beck, P. 2019, Meteor. Planet. Sci., 54, 1951

Iwata, T., Kitazato, K., Abe, M., et al. 2017, Space Sci. Rev., 208, 317

Kitazato, K., Milliken, R. E., Iwata, T., et al. 2019, Science, 364, 272

Li, S., \& Milliken, R. E. 2017, Sci. Adv., 3, e1701471

Milliken, R. E., \& Mustard, J. F. 2005, J. Geophys. Res., 110, E12001

Milliken, R. E., \& Mustard, J. F. 2007a, Icarus, 189, 550

Milliken, R. E., \& Mustard, J. F. 2007b, Icarus, 189, 574

Milliken, R. E., Mustard, J. F., Poulet, F., et al. 2007, J. Geophys. Res., 112, E08S07

Mustard, J. F., Murchie, S. L., Pelkey, S. M., et al. 2008, Nature, 454, 305

Piani, L., Marrocchi, Y., Rigautier, T., et al. 2020, Science, 369, 1110

Pommerol, A., \& Schmitt, B. 2008a, J. Geophys. Res. Planets, 113, E12

Pommerol, A., \& Schmitt, B. 2008b, J. Geophys. Res. Planets, 113, E10

Potin, S., Beck, P., Schmitt, B., \& Moynier, F. 2019, Icarus, 333, 415

Praet, A., Barucci, M. A., Clark, B. E., et al. 2021, Icarus, 363, 114427

Sugita, S., Honda, R., Morota, R., et al. 2019, Science, 364, 252

Takir, D., Emery, J. P., McSween, H. Y., et al. 2013, Meteorit. Planet. Sci., 48, 1618

Takir, D., Stockstill-Cahill, K. R., Hibbitts, C. A., \& Nakauchi, Y. 2019, Icarus, 333,243

Watanabe, S., Hirabayashi, M., Hirata, N., et al. 2019, Science, 364, 268 


\section{Appendix A: Meteorites used for the analysis}

Table A.1. Selected meteorites used in this study reported with their spectral reference (in the last column).

\begin{tabular}{|c|c|c|c|c|c|c|}
\hline Meteorite names & Meteorite types & $\begin{array}{c}\mathrm{H} \text { content } \pm \mathrm{H} \text { error }(\mathrm{wt} . \%) \\
\text { (Reference) }\end{array}$ & $\begin{array}{c}\text { ESPAT } \\
\text { (normalized unit) }\end{array}$ & $\begin{array}{c}\text { NOPL } \\
\text { (normalized unit) }\end{array}$ & $\begin{array}{l}\lambda \text { continuum } \\
(\mu \mathrm{m})\end{array}$ & Spectral reference \\
\hline ALH83100 & $\mathrm{CM} 1 / 2$ & $1.353 \pm 0.009(a)$ & 0.644 & 0.477 & $2.64-3.34$ & Garenne et al. (2016) \\
\hline ALH83100 & $\mathrm{CM} 1 / 2$ & $1.353 \pm 0.009(a)$ & 0.559 & 0.416 & $2.64-3.33$ & Potin et al. (2019) \\
\hline ALH84029 & $\mathrm{CM} 1 / 2$ & $1.260 \pm 0.009(a)$ & 0.694 & 0.488 & $2.64-3.32$ & Garenne et al. (2016) \\
\hline ALH84033 & Heated CM & $0.610 \pm 0.018(a)$ & 0.160 & 0.205 & $2.66-3.34$ & Garenne et al. (2016) \\
\hline ALH84044 & $\mathrm{CM} 1 / 2$ & $1.240 \pm 0.008(a)$ & 0.427 & 0.394 & $2.66-3.32$ & Garenne et al. (2016) \\
\hline Allende & CV3 & $0.09 \pm 0.000(a)$ & 0.120 & 0.143 & $2.66-3.30$ & Takir et al. (2019) \\
\hline Banten & CM2 & $0.910 \pm 0.01(a)$ & 0.045 & 0.064 & $2.68-3.34$ & Takir et al. (2019) \\
\hline Cold Bokkeveld & CM2 & $1.220 \pm 0.004(a)$ & 0.302 & 0.264 & $2.63-3.30$ & Takir et al. (2013) \\
\hline DOM03183 & Heated CM & $0.950 \pm 0.001(a)$ & 0.196 & 0.212 & $2.62-3.32$ & Garenne et al. (2016) \\
\hline DOM08003 & $\mathrm{CM} 2$ & $1.359 \pm 0.006(a)$ & 0.538 & 0.403 & $2.62-3.32$ & Potin et al. (2019) \\
\hline EET83355 & Heated CM & $0.410 \pm 0.001(a)$ & 0.110 & 0.144 & $2.64-3.32$ & Garenne et al. (2016) \\
\hline EET96029 & Heated CM & $0.728 \pm 0.012(a)$ & 0.197 & 0.239 & $2.66-3.34$ & Garenne et al. (2016) \\
\hline EET96029 & Heated CM & $0.728 \pm 0.012(a)$ & 0.099 & 0.106 & $2.63-3.34$ & Potin et al. (2019) \\
\hline Essebi & CM2? & $0.770 \pm 0.005(a)$ & 0.254 & 0.243 & $2.66-3.31$ & Takir et al. (2019) \\
\hline GRA06100 & Heated CR & $0.13 \pm 0.003(a)$ & 0.085 & 0.124 & $2.66-3.32$ & Garenne et al. (2016) \\
\hline Ivuna & CI1 & $1.330 \pm 0.094(a)$ & 0.551 & 0.394 & $2.60-3.25$ & Takir et al. (2013) \\
\hline LAP02277 & Heated CM & $1.112 \pm 0.009(a)$ & 0.393 & 0.317 & $2.66-3.10$ & Takir et al. (2013) \\
\hline LAP02333 & $\mathrm{CM} 2$ & $1.020 \pm 0.005(a)$ & 0.186 & 0.220 & $2.66-3.32$ & Garenne et al. (2016) \\
\hline LAP02336 & CM2 & $0.950 \pm 0.005(a)$ & 0.226 & 0.251 & $2.64-3.34$ & Garenne et al. (2016) \\
\hline LEW87022 & CM2 & $1.090 \pm 0.014(a)$ & 0.293 & 0.295 & $2.64-3.32$ & Garenne et al. (2016) \\
\hline LEW90500 & CM2 & $0.990 \pm 0.005(a)$ & 0.403 & 0.361 & $2.62-3.34$ & Garenne et al. (2016) \\
\hline MAC88100 & Heated CM & $0.894 \pm 0.010(a)$ & 0.321 & 0.281 & $2.64-3.34$ & Potin et al. (2019) \\
\hline MET01070 & CM1 & $1.274 \pm 0.000(a)$ & 0.535 & 0.405 & $2.64-3.32$ & Garenne et al. (2016) \\
\hline MET01070 & CM1 & $1.274 \pm 0.000(a)$ & 0.333 & 0.280 & $2.64-3.34$ & Potin et al. (2019) \\
\hline Mighei & CM2 & $0.994 \pm 0.003(a)$ & 0.220 & 0.240 & $2.66-3.33$ & G. Poggiali, (priv. comm. \\
\hline MIL07700 & Heated CM & $0.570 \pm 0.016(a)$ & 0.236 & 0.251 & $2.64-3.33$ & Garenne et al. (2016) \\
\hline MIL07700 & Heated CM & $0.570 \pm 0.016(a)$ & 0.091 & 0.096 & $2.65-3.33$ & Potin et al. (2019) \\
\hline MIL07700 & Heated CM & $0.570 \pm 0.016(a)$ & 0.011 & 0.013 & $2.71-3.10$ & Takir et al. (2013) \\
\hline Murchison & $\mathrm{CM} 2$ & $0.956 \pm 0.002(a)$ & 0.117 & 0.130 & $2.65-3.32$ & Potin et al. (2019) \\
\hline Murchison & CM2 & $0.956 \pm 0.002(a)$ & 0.357 & 0.287 & $2.62-3.34$ & Takir et al. (2019) \\
\hline Orgueil BM & CI1 & $1.366 \pm 0.015(a)$ & 0.547 & 0.399 & $2.66-3.33$ & Potin et al. (2019) \\
\hline Orgueil Smith. & CI1 & $1.346 \pm 0.019(a)$ & 0.547 & 0.399 & $2.66-3.33$ & Potin et al. (2019) \\
\hline Orgueil BM & CI1 & $1.366 \pm 0.015(a)$ & 0.532 & 0.395 & $2.64-3.33$ & Takir et al. (2019) \\
\hline Orgueil Smith. & CI1 & $1.346 \pm 0.019(a)$ & 0.532 & 0.395 & $2.64-3.33$ & Takir et al. (2019) \\
\hline QUE97990 & $\mathrm{CM} 2$ & $0.959 \pm 0.000(a)$ & 0.066 & 0.077 & $2.67-3.33$ & Potin et al. (2019) \\
\hline QUE97990 & $\mathrm{CM} 2$ & $0.959 \pm 0.000(a)$ & 0.148 & 0.159 & $2.60-3.34$ & Takir et al. (2013) \\
\hline QUE99038 & $\mathrm{CV}$ & $0.06 \pm 0.001(a)$ & 0.006 & 0.009 & $2.71-3.14$ & Takir et al. (2013) \\
\hline RBT04133 & CR & $0.26 \pm 0.001(a)$ & 0.106 & 0.151 & $2.64-3.32$ & Garenne et al. (2016) \\
\hline TL4 & $\mathrm{C} 2$ & $0.72 \pm 0.000(c)$ & 0.098 & 0.101 & $2.65-3.31$ & Potin et al. (2019) \\
\hline TL5b & $\mathrm{C} 2$ & $0.72 \pm 0.003(a)$ & 0.229 & 0.199 & $2.60-3.30$ & Takir et al. (2019) \\
\hline TL11 & $\mathrm{C} 2$ & $0.642 \pm 0.004(a)$ & 0.229 & 0.199 & $2.60-3.30$ & Takir et al. (2019) \\
\hline WIS91600 & Heated CM & $0.878 \pm 0.000(b)$ & 0.239 & 0.248 & $2.64-3.30$ & Garenne et al. (2016) \\
\hline WIS91600 & Heated CM & $0.878 \pm 0.000(b)$ & 0.066 & 0.070 & $2.65-3.30$ & Potin et al. (2019) \\
\hline
\end{tabular}

Notes. The H content is from (a) Alexander et al. (2012, 2013), (b) Garenne et al. (2016), and (c): Gilmour et al. (2019). Their ESPAT and NOPL values, both calculated at the wavelength center of $2.72 \mu \mathrm{m}$, are reported, as are the used continuum anchor point wavelengths $(\lambda)$. As for grain size information, the spectra from Takir et al. 2013, 2019 were taken from a meteorite sample ground into $100 \mu \mathrm{m}$ powder, with no grain size distribution determined due to meteorite sample scarcity. The meteorite reflectance spectra from Potin et al. (2019) and Garenne et al. (2016) were measured on manually ground, non-sieved samples to retain a large grain size distribution. Finally, the Mighei spectrum was measured at INAF-Astrophysical Observatory of Arcetri in Firenze (Italy) on a powder sample with grain size $<500 \mu m$ (Poggiali, priv. comm.). 